\title{
Circulant preconditioners for analytic functions of Toeplitz matrices
}

\author{
Sean Hon • Andrew Wathen
}

Received: date / Accepted: date

\begin{abstract}
Circulant preconditioning for symmetric Toeplitz systems has been well developed over the past few decades. For a large class of such systems, descriptive bounds on convergence for the conjugate gradient method can be obtained. For (real) nonsymmetric Toeplitz systems, much work had been focused on normalising the original systems until [J. Pestana and A. J. Wathen. SIAM J. MATRIX ANAL. APPL. Vol. 36, No. 1, pp. 273-288] recently showed that theoretic guarantees on convergence for the minimal residual method can be established via the simple use of reordering. The authors further proved that a suitable absolute value circulant preconditioner can be used to ensure rapid convergence. In this paper, we show that the related ideas can also be applied to the systems defined by analytic functions of (real) nonsymmetric Toeplitz matrices. For the systems defined by analytic functions of non-Hermitian Toeplitz matrices, we also show that certain circulant preconditioners are effective. Numerical examples with the conjugate gradient method and the minimal residual method are given to support our theoretical results.
\end{abstract}

Keywords Toeplitz matrices · Functions of matrices · Circulant preconditioners $\cdot$ PCG $\cdot$ PMINRES

Mathematics Subject Classification (2000) 65F08 - 65F10 - 65F15 . $15 \mathrm{~A} 16 \cdot 15 \mathrm{~B} 05$

Sean Hon

Mathematical Institute, University of Oxford, Radcliffe Observatory Quarter, Oxford, OX2 6GG, United Kingdom

E-mail: hon@maths.ox.ac.uk

Andrew Wathen

Mathematical Institute, University of Oxford, Radcliffe Observatory Quarter, Oxford, OX2 6GG, United Kingdom

E-mail: wathen@maths.ox.ac.uk 


\section{Introduction}

Instead of directly dealing with the $n \times n$ (real) nonsymmetric Toeplitz system $A_{n} \mathbf{x}=\mathbf{b}$, Pestana and Wathen suggested in [14,11 that one can pre(or post)multiply by the "flip" matrix $Y_{n}$, defined as

$$
Y_{n}=\left[\begin{array}{l}
1 \\
1
\end{array} . \cdot \in \mathbb{R}^{n \times n}\right.
$$

to obtain the symmetric system $Y_{n} A_{n} \mathbf{x}=Y_{n} \mathbf{b}$. Using a suitable absolute value circulant matrix $\left|C_{n}\right|$ as a preconditioner in which $C_{n}$ is derived from $A_{n}$ in a standard way, they further proved that the eigenvalues of the preconditioned matrix $\left|C_{n}\right|^{-1} Y_{n} A_{n}$ are clustered at \pm 1 . The absolute value circulant matrix is readily computed from the diagonalisation provided by a Fast Fourier Transform. One can then use the minimal residual method (MINRES) for solving such symmetric but possibly indefinite system with a guarantee of convergence which depends only on its spectrum.

In this work, we show that Pestana and Wathen's idea of using $Y_{n}$ as a reordering device and $\left|C_{n}\right|$ as a preconditioner can both also be applied to the systems defined by analytic functions of (real) nonsymmetric Toeplitz matrices, i.e. systems of the form $h\left(A_{n}\right) \mathbf{x}=\mathbf{b}$. Note that $h\left(A_{n}\right)$ is generally not Toeplitz. Based on those ideas, we show that one can solve the symmetric system $Y_{n} h\left(A_{n}\right) \mathbf{x}=Y_{n} \mathbf{b}$ using MINRES with a guarantee of convergence which depends only the eigenvalues of $Y_{n} h\left(A_{n}\right)$ instead of solving its normal equation system. In particular, we also show that $\left|h\left(C_{n}\right)\right|^{-1} Y_{n} h\left(A_{n}\right)$ can be decomposed into the sum of a unitary matrix, a matrix of small norm and a matrix of low rank under suitable assumptions.

Our previous work [8] showed that $g\left(c\left(A_{n}\right)\right)$ could be an effective preconditioner for $g\left(A_{n}\right)$, where $g(z)$ is the trigonometric function $e^{z}, \sin z$ or $\cos z$, $A_{n}$ is the Toeplitz matrix generated by a continuous complex-valued function defined on $[-\pi, \pi]$ and $c\left(A_{n}\right)$ is the optimal circulant preconditioner [4] for $A_{n}$. In this work, instead of assuming a trigonometric function, we extend it to the larger class of analytic functions.

Functions of Toeplitz matrices have some crucial applications. For example, $e^{A_{n}}$ arises from the discretisation of integro-differential equations with a shift-invariant kernel 9 . Solving those equations is often required in areas such as the option pricing [5, 15]. Related work on computing the exponential of a block Toeplitz matrix arising in approximations of Markovian fluid queues can also be found in [2]. As for the matrix cosine and sine functions, an example of applications is solving the following system of second order differential equations [7]

$$
\frac{d}{d t^{2}} y+A_{n}^{2} y=0, y(0)=y_{0}, y^{\prime}(0)=y_{0}^{\prime}
$$


whose solution is given by

$$
y(t)=\cos \left(t A_{n}\right) y_{0}+A_{n}^{-1} \sin \left(t A_{n}\right) y_{0}^{\prime}
$$

It is noted that $\left|h\left(C_{n}\right)\right|$ and $h\left(C_{n}\right)$ are circulant matrices. By the diagonalisation of a circulant matrix $C_{n}=U_{n}^{*} \Lambda_{n} U_{n}$, where $U_{n}$ is a Fourier matrix in which the entries are given by $\left[U_{n}\right]_{j k}=\frac{1}{\sqrt{n}} e^{-2 \pi \mathbf{i} j k / n}$ with $j, k=0,1, \ldots, n-1$, we have $\left|h\left(C_{n}\right)\right|=U_{n}^{*}\left|h\left(\Lambda_{n}\right)\right| U_{n}$ and $h\left(C_{n}\right)=U_{n}^{*} h\left(\Lambda_{n}\right) U_{n}$. Therefore, for any vector $\mathbf{d}$ the products $\left|h\left(C_{n}\right)\right|^{-1} \mathbf{d}$ and $h\left(C_{n}\right)^{-1} \mathbf{d}$ can be efficiently computed by Fast Fourier Transforms in $\mathcal{O}(n \log n)$ operations.

It must be noted however that fast matrix vector multiplication with the matrix $h\left(A_{n}\right)$ is not readily archived by circulant embedding, as for the simple case $h(z)=z$, though sparsity may still help. Indeed for $e^{A_{n}}$, the matrix vector multiplication can be computed efficiently for example by a fast algorithm in [10].

\section{Preliminary results on $c\left(A_{n}\right)$ and $A_{n}$}

In the context of iterative solvers for Toeplitz systems, we assume that the given $n \times n$ Toeplitz matrix $A_{n}$ is associated with the function $f$ via its Fourier series

$$
f(\theta)=\sum_{k=-\infty}^{\infty} a_{k} e^{i k \theta}
$$

defined on $[-\pi, \pi]$. We have

$$
A_{n}=\left[\begin{array}{ccccc}
a_{0} & a_{-1} & \cdots & a_{-n+2} & a_{-n+1} \\
a_{1} & a_{0} & a_{-1} & & a_{-n+2} \\
\vdots & a_{1} & a_{0} & \ddots & \vdots \\
a_{n-2} & & \ddots & \ddots & a_{-1} \\
a_{n-1} & a_{n-2} & \cdots & a_{1} & a_{0}
\end{array}\right]
$$

where

$$
a_{k}=\frac{1}{2 \pi} \int_{-\pi}^{\pi} f(\theta) e^{-\mathbf{i} k \theta} d \theta, \quad k=0, \pm 1, \pm 2, \ldots,
$$

are the Fourier coefficients of $f$. The function $f$ is called the generating function of the Toeplitz matrix. If $f$ is complex-valued, $A_{n}$ is non-Hermitian for all $n$. If $f$ is real-valued, $A_{n}$ is Hermitian for all $n$. If $f$ is real-valued and positive, $A_{n}$ is Hermitian positive definite for all $n$. If $f$ is real-valued and even, $A_{n}$ is symmetric for all $n[12$.

We first provide some lemmas concerning Toeplitz matrices. Let $\mathcal{C}[-\pi, \pi]$ be the Banach space of continuous complex-valued functions defined on $[-\pi, \pi]$ with the supremum norm $\|\cdot\|_{\infty}$. 
Lemma 1 [3, Lemma 1 and 3] Let $f \in \mathcal{C}[-\pi, \pi]$. Let $A_{n} \in \mathbb{C}^{n \times n}$ be the Toeplitz matrix generated by $f$ and $c\left(A_{n}\right) \in \mathbb{C}^{n \times n}$ be the optimal circulant preconditioner for $A_{n}$, i.e. $c\left(A_{n}\right)=\min _{W_{n} \in \mathcal{M}_{U_{n}}}\left\|A_{n}-W_{n}\right\|_{F}$ where $\mathcal{M}_{U_{n}}$ is the set of all circulant matrices. Then we have

$$
\left\|A_{n}\right\|_{2} \leq 2\|f\|_{\infty} \text { and }\left\|c\left(A_{n}\right)\right\|_{2} \leq 2\|f\|_{\infty} \quad n=1,2, \ldots
$$

Lemma 2 [3, Theorem 1] Let $f \in \mathcal{C}[-\pi, \pi]$. Let $A_{n}$ be the Toeplitz matrix generated by $f$ and $c\left(A_{n}\right)$ be the optimal circulant preconditioner for $A_{n}$. Then for all $\epsilon>0$ there exist positive integers $N$ and $M>0$ such that for all $n>N$ we have

$$
c\left(A_{n}\right)-A_{n}=V_{n}-W_{n}
$$

where

$$
\operatorname{rank} V_{n} \leq 2 M
$$

and

$$
\left\|W_{n}\right\|_{2} \leq \epsilon
$$

\section{The spectra of the preconditioned matrices}

In this section, we first provide several lemmas concerning functions of matrices.

Lemma 3 [6. Theorem 1.18] Let $h(z)$ be analytic on an open subset $\Omega \subseteq \mathbb{C}$ such that each connected component of $\Omega$ is closed under conjugation. Consider the corresponding matrix function $h(z)$ on its natural domain in $\mathbb{C}^{n \times n}$, the set $\mathcal{D}=\left\{A_{n} \in \mathbb{C}^{n \times n}: \Lambda\left(A_{n}\right) \subseteq \Omega\right\}$. Then the following are equivalent:

(a) $h\left(A_{n}^{*}\right)=h\left(A_{n}\right)^{*}$ for all $A_{n} \in \mathcal{D}$.

(b) $h\left(\overline{A_{n}}\right)=\overline{h\left(A_{n}\right)}$ for all $A_{n} \in \mathcal{D}$.

(c) $h\left(\mathbb{R}^{n \times n} \cap \mathcal{D}\right) \subseteq \mathbb{R}^{n \times n}$

(d) $h(\mathbb{R} \cap \Omega) \subseteq \mathbb{R}$.

Lemma 4 [6, Theorem 4.7] Suppose $h(z)$ has a Taylor series expansion

$$
h(z)=\sum_{k=0}^{\infty} a_{k}(z-\alpha)^{k},
$$

where $a_{k}=\frac{h^{(k)}(\alpha)}{k !}$, with radius of convergence $r$. If $A_{n} \in \mathbb{C}^{n \times n}$ then $h\left(A_{n}\right)$ is defined and is given by

$$
h\left(A_{n}\right)=\sum_{k=0}^{\infty} a_{k}\left(A_{n}-\alpha I_{n}\right)^{k}
$$

if and only if the distinct eigenvalues $\lambda_{1}, \cdots, \lambda_{s}$ of $A_{n}$ satisfies one of the conditions

(a) $\left|\lambda_{i}-\alpha\right|<r$

(b) $\left|\lambda_{i}-\alpha\right|=r$ and the series for $h^{\left(n_{i}-1\right)}(\lambda)$, where $n_{i}$ is the index of $\lambda_{i}$, is convergent at the point $\lambda=\lambda_{i}, i=1, \ldots, s$. 
Lemma 5 [6, Theorem 4.8] Suppose $h(z)$ has a Taylor series expansion

$$
h(z)=\sum_{k=0}^{\infty} a_{k}(z-\alpha)^{k}
$$

where $a_{k}=\frac{h^{(k)}(\alpha)}{k !}$, with radius of convergence $r$. If $A_{n} \in \mathbb{C}^{n \times n}$ with $\rho\left(A_{n}\right.$ $\left.\alpha I_{n}\right)<r$ then for any matrix norm $\|\cdot\|$

$\left\|h\left(A_{n}\right)-\sum_{k=0}^{K-1} a_{k}\left(A_{n}-\alpha I_{n}\right)^{k}\right\| \leq \frac{1}{K !} \max _{0 \leq t \leq 1}\left\|\left(A_{n}-\alpha I_{n}\right)^{K} h^{(K)}\left(\alpha I_{n}+t\left(A_{n}-\alpha I_{n}\right)\right)\right\|$.

The following lemma shows that $Y_{n} h\left(A_{n}\right)$ is (real) symmetric when $A_{n}$ is a (real) nonsymmetric Toeplitz matrix.

Lemma 6 Given $h(z)$ is analytic on $|z|<r$. If $A_{n} \in \mathbb{R}^{n \times n}$ with $\rho\left(A_{n}\right)<r$ is (real) persymmetric, i.e. $Y_{n} A_{n}=A_{n}^{T} Y_{n}$, then $h\left(A_{n}\right)$ is also (real) persymmetric.

Proof We start by showing that $A_{n}^{k}$ is (real) persymmetric for any nonnegative integer $k$ :

$$
\begin{aligned}
Y_{n} A^{k} & =Y_{n} A_{n} A_{n}^{k-1} \\
& =A_{n}^{T} Y_{n} A_{n}^{k-1} \\
& =A_{n}^{T} Y_{n} A_{n} A_{n}^{k-2} \\
& =\left(A_{n}^{T}\right)^{2} Y_{n} A_{n}^{k-2} \\
& \vdots \\
& =\left(A_{n}^{T}\right)^{k} Y_{n} \\
& =\left(A_{n}^{k}\right)^{T} Y_{n} .
\end{aligned}
$$

Since $h(z)$ is analytic on $|z|<r$, it has the following Taylor series representation:

$$
h(z)=\sum_{k=0}^{\infty} a_{k} z^{k}
$$

with the radius of convergence $r$. By Lemma 4 , we have

$$
h\left(A_{n}\right)=\sum_{k=0}^{\infty} a_{k} A_{n}^{k}
$$


Thus,

$$
\begin{aligned}
Y_{n} h\left(A_{n}\right) & =Y_{n} \lim _{K \rightarrow \infty} \sum_{k=0}^{K} a_{k} A_{n}^{k} \\
& =\lim _{K \rightarrow \infty} \sum_{k=0}^{K} a_{k} Y_{n} A_{n}^{k} \\
& =\lim _{K \rightarrow \infty} \sum_{k=0}^{K} a_{k}\left(A_{n}^{k}\right)^{T} Y_{n} \\
& =\lim _{K \rightarrow \infty}\left(\sum_{k=0}^{K} a_{k}\left(A_{n}^{k}\right)\right)^{T} Y_{n} \\
& =h\left(A_{n}\right)^{T} Y_{n} .
\end{aligned}
$$

Moreover, by Lemma $3(\mathrm{c}), h\left(A_{n}\right)$ is real when $A_{n}$ is real. The result follows.

We are now ready to give our main results on the spectrum of $\left|h\left(c\left(A_{n}\right)\right)\right|^{-1} h\left(A_{n}\right)$. Without loss of generality, we assume that $h(z)$ is represented by the following Taylor series:

$$
h(z)=\sum_{k=0}^{\infty} a_{k} z^{k} .
$$

Theorem 1 Given $h(z)$ is analytic on $|z|<r$. Let $f \in \mathcal{C}[-\pi, \pi]$ with $2\|f\|_{\infty}<$ $r$. Let $A_{n}$ be the Toeplitz matrix generated by $f$ and $c\left(A_{n}\right)$ be the optimal circulant preconditioner for $A_{n}$. Then, for all $\epsilon>0$ there exist integers $N$ and $M$ such that for all $n>N$

$$
h\left(c\left(A_{n}\right)\right)-h\left(A_{n}\right)=R_{n}+E_{n},
$$

where

$$
\begin{gathered}
\operatorname{rank} R_{n} \leq 2 M, \\
\left\|E_{n}\right\|_{2} \leq \epsilon .
\end{gathered}
$$

Proof Since $h(z)$ is analytic on $|z|<r$, it has the following Taylor series representation:

$$
h(z)=\sum_{k=0}^{\infty} a_{k} z^{k}
$$

with the radius of convergence $r=\left(\lim _{k \rightarrow \infty}\left|\frac{a_{k+1}}{a_{k}}\right|\right)^{-1}$. By the assumption $2\|f\|_{\infty}<r$ and Lemma 1, we have

$$
\begin{aligned}
r & >2\|f\|_{\infty} \\
& >\left\|A_{n}\right\|_{2} \\
& >\rho\left(A_{n}\right) \\
& =\max _{i} \lambda_{i}\left(A_{n}\right) \\
& \geq \lambda_{i}\left(A_{n}\right) \quad \text { for } \quad i=1,2, \ldots, n,
\end{aligned}
$$


where $\lambda_{i}\left(A_{n}\right)$ denotes the $i$-th eigenvalue of $A_{n}$. Therefore, by Lemma 4 , we have

$$
h\left(A_{n}\right)=\sum_{k=0}^{\infty} a_{k} A_{n}^{k} .
$$

Similarly, we have

$$
h\left(c\left(A_{n}\right)\right)=\sum_{k=0}^{\infty} a_{k} c\left(A_{n}\right)^{k} .
$$

We now decompose

$$
\begin{aligned}
& h\left(c\left(A_{n}\right)\right)-h\left(A_{n}\right) \\
= & \underbrace{h\left(c\left(A_{n}\right)\right)-\sum_{k=0}^{K} a_{k} c\left(A_{n}\right)^{k}}_{G_{1}}+\underbrace{\sum_{k=0}^{K} a_{k} c\left(A_{n}\right)^{k}-\sum_{k=0}^{K} a_{k} A_{n}^{k}}_{B}+\underbrace{\sum_{k=0}^{K} a_{k} A_{n}^{k}-h\left(A_{n}\right)}_{G_{2}} .
\end{aligned}
$$

We first get the measure of $\left\|G_{1}+G_{2}\right\|_{2}$. Using Lemmas 5 , we have

$$
\begin{aligned}
& \left\|G_{1}+G_{2}\right\|_{2} \\
\leq & \left\|h\left(c\left(A_{n}\right)\right)-\sum_{k=0}^{K} a_{k} c\left(A_{n}\right)^{k}\right\|_{2}+\left\|h\left(A_{n}\right)-\sum_{k=0}^{K} a_{k} A_{n}^{k}\right\|_{2} \\
\leq & \frac{\max _{0 \leq t \leq 1}\left\|c\left(A_{n}\right)^{K+1} h^{(k+1)}\left(t c\left(A_{n}\right)\right)\right\|_{2}}{(K+1) !}+\frac{\max _{0 \leq t \leq 1}\left\|A_{n}^{K+1} h^{(k+1)}\left(t A_{n}\right)\right\|_{2}}{(K+1) !} \\
\leq & \frac{\left\|c\left(A_{n}\right)^{K+1}\right\|_{2}}{(K+1) !} \max _{0 \leq t \leq 1}\left\|h^{(k+1)}\left(t c\left(A_{n}\right)\right)\right\|_{2}+\frac{\left\|A_{n}^{K+1}\right\|_{2}}{(K+1) !} \max _{0 \leq t \leq 1}\left\|h^{(k+1)}\left(t A_{n}\right)\right\|_{2}
\end{aligned}
$$

Now, by Lemma 1 .

$$
\begin{aligned}
\max _{0 \leq t \leq 1}\left\|h^{(k+1)}\left(t A_{n}\right)\right\|_{2} & =\max _{0 \leq t \leq 1}\left\|\sum_{k=0}^{\infty} \frac{(K+k+1) !}{k !} a_{K+k+1}\left(t A_{n}\right)^{k}\right\|_{2} \\
& \leq \max _{0 \leq t \leq 1} \sum_{k=0}^{\infty} \frac{(K+k+1) !}{k !}\left|a_{K+k+1}\right|\left\|t A_{n}\right\|_{2}^{k} \\
& \leq \sum_{k=0}^{\infty} \frac{(K+k+1) !}{k !}\left|a_{K+k+1}\right|\left\|A_{n}\right\|_{2}^{k} \\
& \leq \sum_{k=0}^{\infty} \frac{(K+k+1) !}{k !}\left|a_{K+k+1}\right|\left(2\|f\|_{\infty}\right)^{k}
\end{aligned}
$$

We now show that $\sum_{k=0}^{\infty} \frac{(K+k+1) !}{k !}\left|a_{K+k+1}\right|\left(2\|f\|_{\infty}\right)^{k}$ is a convergent series using the ratio test. By the assumption $2\|f\|_{\infty}<r=\left(\lim _{k \rightarrow \infty}\left|\frac{a_{k+1}}{a_{k}}\right|\right)^{-1}$, we 
know that

$$
\begin{aligned}
\lim _{k \rightarrow \infty}\left|\frac{a_{K+k+2}}{a_{K+k+1}}\right|\left(\frac{K+k+2}{k+1}\right)\left(2\|f\|_{\infty}\right) & =\lim _{k \rightarrow \infty}\left|\frac{a_{k+1}}{a_{k}}\right|\left(2\|f\|_{\infty}\right) \\
& <\left(\frac{1}{r}\right)\left(2\|f\|_{\infty}\right)
\end{aligned}
$$

which is a constant less than 1 because $2\|f\|_{\infty}<r$.

Therefore, by the ratio test, $\sum_{k=0}^{\infty} \frac{(K+k+1) !}{k !}\left|a_{K+k+1}\right|\left(2\|f\|_{\infty}\right)^{k}$ is convergent. We conclude that $\sum_{k=0}^{\infty} \frac{(K+k+1) !}{k !}\left|a_{K+k+1}\right|\left(2\|f\|_{\infty}\right)^{k}:=m_{\left(2\|f\|_{\infty}\right)}$ is independent of $n$.

Hence,

$$
\max _{0 \leq t \leq 1}\left\|h^{(k+1)}\left(t A_{n}\right)\right\|_{2} \leq m_{\left(2\|f\|_{\infty}\right)}
$$

Similarly, we get

$$
\max _{0 \leq t \leq 1}\left\|h^{(k+1)}\left(\operatorname{tc}\left(A_{n}\right)\right)\right\|_{2} \leq m_{\left(2\|f\|_{\infty}\right)}
$$

Therefore,

$$
\begin{aligned}
& \left\|G_{1}+G_{2}\right\|_{2} \\
\leq & \frac{\left\|c\left(A_{n}\right)^{K+1}\right\|_{2}}{(K+1) !} \max _{0 \leq t \leq 1}\left\|h^{(k+1)}\left(t c\left(A_{n}\right)\right)\right\|_{2}+\frac{\left\|A_{n}^{K+1}\right\|_{2}}{(K+1) !} \max _{0 \leq t \leq 1}\left\|h^{(k+1)}\left(t A_{n}\right)\right\|_{2} \\
\leq & \frac{\left\|c\left(A_{n}\right)\right\|_{2}^{K+1}}{(K+1) !} m_{\left(2\|f\|_{\infty}\right)}+\frac{\left\|A_{n}\right\|_{2}^{K+1}}{(K+1) !} m_{\left(2\|f\|_{\infty}\right)} \\
\leq & \frac{\left(2\|f\|_{\infty}\right)^{K+1}}{(K+1) !} m_{\left(2\|f\|_{\infty}\right)}+\frac{\left(2\|f\|_{\infty}\right)^{K+1}}{(K+1) !} m_{\left(2\|f\|_{\infty}\right)} \\
= & \frac{\left(2\|f\|_{\infty}\right)^{K+1}}{(K+1) !}\left(2 m_{\left(2\|f\|_{\infty}\right)}\right):=\epsilon_{K}
\end{aligned}
$$

which converges to zero as $K$ goes to infinity. Therefore, for a given $\epsilon_{K}>0$, there exists an integer $K$ such that for all $k>K$,

$$
\left\|G_{1}+G_{2}\right\|_{2} \leq \epsilon_{K}
$$

where $k$ is the number of terms in the Taylor series of $c\left(A_{n}\right)$ (or $A_{n}$ ).

We next show that $B$ can be decomposed into a sum of a matrix of fixed rank and a matrix of small norm. Firstly, by Lemma 2, for all $\epsilon>0$ there exist integers $N_{1}$ and $M_{2}>0$ such that for all $n>N_{1}$, we have

$$
c\left(A_{n}\right)-A_{n}=V_{n}-W_{n}
$$


where

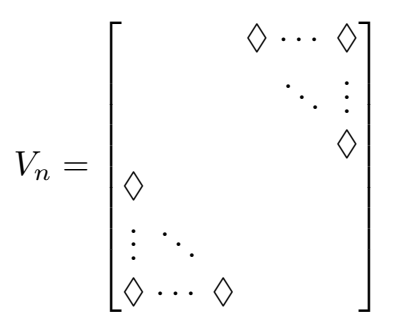

with diamonds representing the non-zero entries,

$\operatorname{rank} V_{n} \leq 2 M_{1}$

and

$$
\left\|W_{n}\right\|_{2} \leq \epsilon
$$

Rewrite $B$ as

$$
\begin{aligned}
& B=\sum_{k=0}^{K} a_{k} c\left(A_{n}\right)^{k}-\sum_{k=0}^{K} a_{k} A_{n}^{k} \\
& =\sum_{k=1}^{K} a_{k}\left(c\left(A_{n}\right)^{k}-A_{n}^{k}\right) \\
& =\sum_{k=1}^{K} a_{k}\left(\sum_{j=0}^{k-1} c\left(A_{n}\right)^{j}\left(c\left(A_{n}\right)-A_{n}\right) A_{n}^{k-1-j}\right) \\
& =\sum_{k=1}^{K} a_{k}\left(\sum_{j=0}^{k-1} c\left(A_{n}\right)^{j}\left(V_{n}-W_{n}\right) A_{n}^{k-1-j}\right) \\
& =\underbrace{\sum_{k=1}^{K} a_{k}\left(\sum_{j=0}^{k-1} c\left(A_{n}\right)^{j} V_{n} A_{n}^{k-1-j}\right)}_{R_{n}}+\underbrace{\sum_{k=1}^{K} a_{k}\left(\sum_{j=0}^{k-1} c\left(A_{n}\right)^{j} W_{n} A_{n}^{k-1-j}\right)}_{J}
\end{aligned}
$$


Using Lemmas 1] we can estimate the norm of $J$ :

$$
\begin{aligned}
\|J\|_{2} & =\left\|\sum_{k=1}^{K} a_{k} \sum_{j=0}^{k-1} c\left(A_{n}\right)^{j} W_{n} A_{n}^{k-1-j}\right\|_{2} \\
& \leq \sum_{k=1}^{K}\left|a_{k}\right|\left\|\sum_{j=0}^{k-1} c\left(A_{n}\right)^{j} W_{n} A_{n}^{k-1-j}\right\|_{2} \\
& \leq\left\|W_{n}\right\|_{2} \sum_{k=1}^{K}\left|a_{k}\right| \sum_{j=0}^{k-1}\left\|c\left(A_{n}\right)\right\|_{2}^{j}\left\|A_{n}\right\|_{2}^{k-1-j} \\
& \leq\left\|W_{n}\right\|_{2} \sum_{k=1}^{K}\left|a_{k}\right| \sum_{j=0}^{k-1}\left(2\|f\|_{\infty}\right)^{j}\left(2\|f\|_{\infty}\right)^{k-1-j} \\
& =\left\|W_{n}\right\|_{2} \underbrace{\sum_{k=1}^{K}\left|a_{k}\right| \sum_{j=0}^{k-1} k\left(2\|f\|_{\infty}\right)^{k-1}}_{m_{0}} \\
& \leq m_{0} \epsilon,
\end{aligned}
$$

where $m_{0}$ is a constant independent of $n$.

We now show that rank $R_{n} \leq 2 K M_{1}$ by first investigating the structure of $R_{n}$. Similar to the approach used in the proof of Lemma 3.11 in [13, simple computations yield

$$
c\left(A_{n}\right)^{\alpha} V_{n} A_{n}^{\beta}=\left[\begin{array}{cccccc}
\diamond & \cdots & \diamond & \diamond & \cdots & \diamond \\
\vdots & \diamond & \vdots & \vdots & \diamond & \vdots \\
\diamond & \cdots & \diamond & \diamond & \cdots & \diamond \\
& & & & \\
\diamond & \cdots & \diamond & \diamond & \cdots & \diamond \\
\vdots & \diamond & \vdots & \vdots & \diamond & \vdots \\
\diamond & \cdots & \diamond & \diamond & \cdots & \diamond
\end{array}\right],
$$

where the diamonds represent the non-zero entries which appear only in the four $(\alpha+1) M_{1}$ by $(\beta+1) M_{1}$ blocks in the corners, provided that $n$ is larger than $2 \max (\alpha+1, \beta+1) M_{1}$. Since the rank of

$$
R_{n}=\sum_{k=1}^{K} a_{i}\left(\sum_{j=0}^{k-1} c\left(A_{n}\right)^{j} V_{n} A_{n}^{k-1-j}\right)
$$

is determined by that of $\sum_{j=0}^{K-1} c\left(A_{n}\right)^{j} V_{n} A_{n}^{K-1-j}$ which is a block matrix with only four non-zero $K M_{1}$ by $K M_{1}$ blocks in its corners, it follows that the rank of $R_{n}$ is less than or equal to $2 K M_{1}$ if we assume $n>2 \underbrace{K M_{1}}_{M}$. 
Hence, we pick

$$
N:=\max \left\{N_{1}, 2 M\right\},
$$

and, combining (1) and (2), it follows that for all $n>N$ we have

$$
\|\underbrace{G_{1}+J+G_{2}}_{E_{n}}\|_{2} \leq m_{0} \epsilon+\epsilon_{K}
$$

The result follows.

Corollary 1 Given $h(z)$ is analytic on $|z|<r$. Let $f \in \mathcal{C}[-\pi, \pi]$ with $2\|f\|_{\infty}<$ $r$. Let $A_{n}$ be the Toeplitz matrix generated by $f$ and $c\left(A_{n}\right)$ be the optimal circulant preconditioner for $A_{n}$. If $\left\|h\left(c\left(A_{n}\right)\right)^{-1}\right\|_{2}$ is bounded for $n=1,2, \ldots$, then for all $\epsilon>0$ there exist positive integers $N$ and $M$ such that for all $n>N$

$$
\left|h\left(c\left(A_{n}\right)\right)\right|^{-1} h\left(A_{n}\right)=Q_{n}+\widehat{R_{n}}+\widehat{E_{n}}
$$

where $Q_{n}$ is unitary,

$$
\begin{gathered}
\operatorname{rank} \widehat{R_{n}} \leq 2 M, \\
\left\|\widehat{E_{n}}\right\|_{2} \leq \epsilon .
\end{gathered}
$$

Proof As $h\left(c\left(A_{n}\right)\right)$ is a circulant matrix we write $h\left(c\left(A_{n}\right)\right)=U_{n}^{*} h\left(\Lambda_{n}\right) U_{n}$ where $h\left(\Lambda_{n}\right)$ is the diagonal matrix with the eigenvalues of $h\left(c\left(A_{n}\right)\right)$. We then have

$$
\begin{aligned}
\left|h\left(c\left(A_{n}\right)\right)\right| & =U_{n}^{*}\left|h\left(\Lambda_{n}\right)\right| U_{n} \\
& =U_{n}^{*} h\left(\Lambda_{n}\right) U_{n} \underbrace{U_{n}^{*} \operatorname{sign}\left(h\left(\Lambda_{n}\right)\right)^{-1} U_{n}}_{Q_{n}} \\
& =h\left(c\left(A_{n}\right)\right) Q_{n},
\end{aligned}
$$

where $\operatorname{sign}\left(h\left(\Lambda_{n}\right)\right)=\operatorname{diag}\left(\frac{h\left(\Lambda_{i}\right)}{\left|h\left(\Lambda_{i}\right)\right|}\right)$, and $Q_{n}$ is unitary.

By Theorem 1, we know that for all $\epsilon>0$, there exist positive integers $N$ and $M$ such that for all $n>N$

$$
h\left(c\left(A_{n}\right)\right)-h\left(A_{n}\right)=R_{n}+E_{n}
$$

where

rank $R_{n} \leq 2 M$

$$
\left\|E_{n}\right\|_{2} \leq \epsilon .
$$

By the assumption that $\left\|h\left(c\left(A_{n}\right)\right)^{-1}\right\|_{2}<m_{0}$ for $n=1,2, \ldots$, where $m$ is a positive constant independent of $n$, we have

$$
\begin{aligned}
h\left(c\left(A_{n}\right)\right)^{-1} h\left(A_{n}\right) & =I_{n}+h\left(c\left(A_{n}\right)\right)^{-1}\left(h\left(A_{n}\right)-h\left(c\left(A_{n}\right)\right)\right) \\
& =I_{n}+h\left(c\left(A_{n}\right)\right)^{-1}\left(-R_{n}\right)+h\left(c\left(A_{n}\right)\right)^{-1}\left(-E_{n}\right) .
\end{aligned}
$$


Further using (4), we have

$$
\begin{aligned}
\left|h\left(c\left(A_{n}\right)\right)\right|^{-1} h\left(A_{n}\right) & =Q_{n} h\left(c\left(A_{n}\right)\right)^{-1} h\left(A_{n}\right) \\
& =Q_{n}+\underbrace{Q_{n} h\left(c\left(A_{n}\right)\right)^{-1}\left(-R_{n}\right)}_{\widehat{R}_{n}}+\underbrace{Q_{n} h\left(c\left(A_{n}\right)\right)^{-1}\left(-E_{n}\right)}_{\widehat{E}_{n}} .
\end{aligned}
$$

Since $Q_{n}$ is unitary, we know

$$
\operatorname{rank}\left(\widehat{R}_{n}\right)=\operatorname{rank}\left(Q_{n} h\left(c\left(A_{n}\right)\right)^{-1} R_{n}\right)=\operatorname{rank}\left(R_{n}\right) \leq 2 M
$$

and

$$
\left\|\widehat{E}_{n}\right\|_{2}=\left\|Q_{n} h\left(c\left(A_{n}\right)\right)^{-1} E_{n}\right\|_{2}=\left\|h\left(c\left(A_{n}\right)\right)^{-1} E_{n}\right\|_{2} \leq m_{0} \epsilon .
$$

The result follows.

Assuming $f$ is a real-valued, by Lemma 3 (a), we know that $h\left(A_{n}\right)$ is Hermitian as $A_{n}$ is Hermitian. Similarly, $h\left(c\left(A_{n}\right)\right)$ is Hermitian as $c\left(A_{n}\right)$ is Hermitian. Hence, we consider the following two cases: when $h\left(A_{n}\right)$ is Hermitian indefinite, MINRES can be used with $\left|h\left(c\left(A_{n}\right)\right)\right|$ as the preconditioner. In the special case where both $h\left(A_{n}\right)$ and $h\left(C_{n}\right)$ are Hermitian positive definite, $\left|h\left(c\left(A_{n}\right)\right)\right|$ becomes $h\left(c\left(A_{n}\right)\right)$ and $\mathrm{CG}$ can be applied.

Corollary 2 Given $h(z)$ is analytic on $|z|<r$. Let $f \in \mathcal{C}[-\pi, \pi]$ with $2\|f\|_{\infty}<$ $r$. Let $A_{n}$ be the Toeplitz matrix generated by $f$ and $c\left(A_{n}\right)$ be the optimal circulant preconditioner for $A_{n}$. If $\left\|h\left(c\left(A_{n}\right)\right)^{-1}\right\|_{2}$ is bounded for $n=1,2, \ldots$, then for all $\epsilon>0$, there exist positive integers $N$ and $M$ such that for all $n>N$

$$
\left[\left|h\left(c\left(A_{n}\right)\right)\right|^{-1} h\left(A_{n}\right)\right]^{*}\left|h\left(c\left(A_{n}\right)\right)\right|^{-1} h\left(A_{n}\right)=I_{n}+\overline{R_{n}}+\overline{E_{n}},
$$

where

$$
\begin{aligned}
\operatorname{rank} \overline{R_{n}} & \leq 4 M, \\
\left\|\overline{E_{n}}\right\|_{2} & \leq \epsilon .
\end{aligned}
$$

Proof By Corollary 1, we know that for all $\epsilon>0$ there exist positive integers $N$ and $M$ such that for all $n>N$

$$
\left|h\left(c\left(A_{n}\right)\right)\right|^{-1} h\left(A_{n}\right)=Q_{n}+\widehat{R_{n}}+\widehat{E_{n}},
$$

where $Q_{n}$ is unitary,

$$
\begin{gathered}
\operatorname{rank} \widehat{R_{n}} \leq M, \\
\left\|\widehat{E_{n}}\right\|_{2} \leq \epsilon .
\end{gathered}
$$

We then have

$$
\begin{aligned}
& {\left[\left|h\left(c\left(A_{n}\right)\right)\right|^{-1} h\left(A_{n}\right)\right]^{*}\left|h\left(c\left(A_{n}\right)\right)\right|^{-1} h\left(A_{n}\right) } \\
= & \left(Q_{n}+\widehat{R_{n}}+\widehat{E_{n}}\right)^{*}\left(Q_{n}+\widehat{R_{n}}+\widehat{E_{n}}\right) \\
= & Q_{n}^{*} Q_{n}+\underbrace{*}_{\widehat{R_{n}}}\left(I_{n}+\widehat{R_{n}}+\widehat{E_{n}}\right)+\left(I_{n}+{\widehat{E_{n}}}^{*}\right) \widehat{R_{n}} \\
& +\underbrace{}_{\overline{E_{n}}+{\widehat{E_{n}}}^{*}+{\widehat{E_{n}}}^{*} \widehat{E_{n}}} \\
= & I_{n}+\overline{R_{n}}+\overline{E_{n}} .
\end{aligned}
$$


It immediately follows that $\operatorname{rank} \overline{R_{n}} \leq 4 M$ and $\left\|\overline{E_{n}}\right\|_{2} \leq \epsilon^{2}+2 \epsilon$.

When $h\left(A_{n}\right)$ is non-Hermitian, we consider its normal equation system as shown in Corollary 2. Since $\left[\left|h\left(c\left(A_{n}\right)\right)\right|^{-1} h\left(A_{n}\right)\right]^{*}\left|h\left(c\left(A_{n}\right)\right)\right|^{-1} h\left(A_{n}\right)$ is Hermitian positive definite, we can $\mathrm{CG}$ in this case.

In the special case when $h\left(A_{n}\right)$ is (real) nonsymmetric, it is not necessary to normalise the original system via the following corollary:

Corollary 3 Given $h(z)$ is analytic on $|z|<r$. Let $f \in \mathcal{C}[-\pi, \pi]$ with real Fourier coefficients and $2\|f\|_{\infty}<r$. Let $A_{n}$ be the real Toeplitz matrix generated by $f$ and $c\left(A_{n}\right)$ be the optimal circulant preconditioner for $A_{n}$. If $\left\|h\left(c\left(A_{n}\right)\right)^{-1}\right\|_{2}$ is bounded for $n=1,2, \ldots$, then for all $\epsilon>0$ there exist positive integers $N$ and $M$ such that for all $n>N$

$$
\left|h\left(c\left(A_{n}\right)\right)\right|^{-1} Y_{n} h\left(A_{n}\right)=Q_{n}+\widehat{R_{n}}+\widehat{E_{n}}
$$

where $Q_{n}$ is orthogonal and (real) symmetric,

$$
\begin{gathered}
\operatorname{rank} \widehat{R_{n}} \leq 2 M, \\
\left\|\widehat{E_{n}}\right\|_{2} \leq \epsilon .
\end{gathered}
$$

Proof As $h\left(c\left(A_{n}\right)\right)$ is a circulant matrix we write $h\left(c\left(A_{n}\right)\right)=U_{n}^{*} h\left(\Lambda_{n}\right) U_{n}$ where $h\left(\Lambda_{n}\right)$ is the diagonal matrix with the eigenvalues of $h\left(c\left(A_{n}\right)\right)$. We then have

$$
\begin{aligned}
\left|h\left(c\left(A_{n}\right)\right)\right| & =U_{n}^{*}\left|h\left(\Lambda_{n}\right)\right| U_{n} \\
& =U_{n}^{*} h\left(\Lambda_{n}\right) U_{n}(\underbrace{U_{n}^{*} \operatorname{sign}\left(h\left(\Lambda_{n}\right)\right) U_{n}}_{\widetilde{C_{n}}})^{-1} \\
& =h\left(c\left(A_{n}\right)\right){\widetilde{C_{n}}}^{-1},
\end{aligned}
$$

where $\operatorname{sign}\left(h\left(\Lambda_{n}\right)\right)=\operatorname{diag}\left(\frac{h\left(\Lambda_{i}\right)}{h\left(\Lambda_{i}\right) \mid}\right)$, and $\widetilde{C_{n}}$ is orthogonal.

By Theorem 1, we know that for all $\epsilon>0$, there exist positive integers $N$ and $M$ such that for all $n>N$

$$
h\left(c\left(A_{n}\right)\right)-h\left(A_{n}\right)=R_{n}+E_{n}
$$

where

$$
\begin{gathered}
\operatorname{rank} R_{n} \leq 2 M, \\
\left\|E_{n}\right\|_{2} \leq \epsilon .
\end{gathered}
$$

By (4) and the assumption that $\left\|h\left(c\left(A_{n}\right)\right)^{-1}\right\|_{2}<m_{0}$ for $n=1,2, \ldots$, where $m_{0}$ is a positive constant independent of $n$, we have

$$
\begin{aligned}
\left|h\left(c\left(A_{n}\right)\right)\right|^{-1} Y_{n} h\left(A_{n}\right) & =Y_{n}\left|h\left(c\left(A_{n}\right)\right)\right|^{-1} h\left(A_{n}\right) \\
& =Y_{n}\left|h\left(c\left(A_{n}\right)\right)\right|^{-1}\left(h\left(c\left(A_{n}\right)\right)-R_{n}-E_{n}\right) \\
& =\underbrace{Y_{n} \widetilde{C_{n}}}_{Q_{n}}+\underbrace{Y_{n}\left|h\left(c\left(A_{n}\right)\right)\right|^{-1}\left(-R_{n}\right)}_{\widehat{R}_{n}}+\underbrace{Y_{n}\left|h\left(c\left(A_{n}\right)\right)\right|^{-1}\left(-E_{n}\right)}_{\widehat{E}_{n}} .
\end{aligned}
$$


As $\widetilde{C_{n}}$ is a real circulant matrix, $Q_{n}=Y_{n} \widetilde{C_{n}}$ is (real) symmetric. Also, as $\widetilde{C_{n}}$ is orthogonal, we can show that $Q_{n}$ is orthogonal:

$$
\begin{aligned}
Q_{n}^{T} Q_{n} & =\left(Y_{n} \widetilde{C_{n}}\right)^{T}\left(Y_{n} \widetilde{C_{n}}\right) \\
& ={\widetilde{C_{n}}}^{T}\left(Y_{n}^{T} Y_{n}\right) \widetilde{C_{n}} \\
& ={\widetilde{C_{n}}}^{T} \widetilde{C_{n}} \\
& =I_{n} .
\end{aligned}
$$

We have

$$
\operatorname{rank}\left(\widehat{R}_{n}\right)=\operatorname{rank}\left(Y_{n}\left|h\left(c\left(A_{n}\right)\right)\right|^{-1} R_{n}\right)=\operatorname{rank}\left(R_{n}\right) \leq 2 M
$$

and

$$
\left\|\widehat{E}_{n}\right\|_{2}=\left\|Y_{n}\left|h\left(c\left(A_{n}\right)\right)\right|^{-1} E_{n}\right\|_{2}=\left\|h\left(c\left(A_{n}\right)\right)^{-1} E_{n}\right\|_{2} \leq m_{0} \epsilon .
$$

The result follows.

In the above results, the condition on the uniform boundedness of $\left\|h\left(c\left(A_{n}\right)\right)^{-1}\right\|_{2}$ has been required. In fact, the validity of this condition depends on the composition of the analytic function $h(z)$ and the generating function $f$ of the Toeplitz matrix $A_{n}$. The following examples illustrate the point.

For $h(z)=\sin z$, from

$$
\left\|\left(\sin c\left(A_{n}\right)\right)^{-1}\right\|_{2}=\max _{i}\left|\frac{1}{\sin \lambda_{i}}\right|
$$

where $\lambda_{i}$ is the $i$-th eigenvalue of $c\left(A_{n}\right)$, we know that $\left\|\left(\sin c_{n}[f]\right)^{-1}\right\|_{2}$ could be arbitrarily large since $\sin \lambda_{i}$ could be close to zero. Therefore, we have needed to assume that $\left\|\left(\sin c\left(A_{n}\right)\right)^{-1}\right\|_{2}$ is bounded for $n=1,2, \ldots$.

As for $h(z)=e^{z}$, the condition always holds as we can easily show that

$$
\left\|\left(e^{c\left(A_{n}\right)}\right)^{-1}\right\|_{2} \leq e^{2\|f\|_{\infty}} \quad n=1,2, \ldots
$$

\section{Extension to multilevel cases}

In this section, we briefly discuss that our results can be extended to the multilevel Toeplitz cases. The related proofs can be done in a similar fashion as in the 1D Toeplitz case. Namely, with the appropriate assumptions, we can show that the preconditioned system defined by an analytic function of a multilevel Toeplitz can be decomposed into the sum of a matrix with small norm and a matrix of low rank for a sufficiently large dimension.

Consider the simple block-Toeplitz-Toeplitz-block (BTTB) matrix as an example, $A_{n, m}=H_{n} \otimes K_{m}$ where $H_{n} \in \mathbb{R}^{n \times n}$ and $K_{m} \in \mathbb{R}^{m \times m}$ are both real Toeplitz. Introducing the matrix $Y_{n, m}=Y_{n} \otimes Y_{m}$, it can be easily shown that $Y_{n, m} A_{n, m}=\left(Y_{n} \otimes Y_{m}\right)\left(H_{n} \otimes K_{m}\right)=\left(Y_{n} H_{n}\right) \otimes\left(Y_{m} K_{m}\right)$. Thus $Y_{n, m} A_{n, m}$ is symmetric as both $Y_{n} H_{n}$ and $Y_{m} K_{m}$ are symmetric. Together with an effective absolute value block-circulant-circulant-block (BCCB) preconditioner, we can 
use MINRES for $Y_{n, m} A_{n, m}$ without considering the normal equations system. The well-known difficulty that the low rank part now has been multiplied by the block dimension is as exactly the same issue in our situation as it does for the more standard well-known situation.

Indeed, this symmetrising technique applies to more general real multilevel Toeplitz matrices since they are in general persymmetric. Consequently, by Lemma 6, analytic functions of real multilevel Toeplitz matrices are also persymmetric. Multiplying these matrices by the matrix $Y_{n}$ with the appropriate dimension can result the modified yet symmetric matrices. Again, MINRES with effective absolute value multilevel circulant preconditioners can be used.

\section{Numerical results}

In this section, we demonstrate the effectiveness of our proposed preconditioner $\left|h\left(c\left(A_{n}\right)\right)\right|$ for $h\left(A_{n}\right) \mathbf{x}=\mathbf{b}$ using CG, MINRES and GMRES. Throughout all numerical tests, $e^{A_{n}}$ is computed by the MATLAB built-in function expm whilst $\sin A_{n}, \cos A_{n}$ and other matrix functions are computed by funm. The vector $\mathbf{b}$ is generated by the function ones $(\mathbf{n}, \mathbf{1})$ and the initial guess is the zero vector. Also, we use the function pcg to solve the Hermitian positive definite systems. For Hermitian indefinite systems, we use the function minres. As a comparison, GMRES is also used for some systems and it is executed by gmres. The stopping criterion used is

$$
\frac{\left\|\mathbf{r}_{j}\right\|_{2}}{\|\mathbf{b}\|_{2}}<10^{-7}
$$

where $\mathbf{r}_{j}$ is the residual vector after $j$ iterations.

Example 1. We first consider a Grcar matrix $A_{n}$, namely

$$
A_{n}=\left[\begin{array}{cccccc}
1 & 1 & 1 & 1 & & \\
-1 & \ddots & \ddots & \ddots & \ddots & \\
& \ddots & \ddots & \ddots & \ddots & 1 \\
& & \ddots & \ddots & \ddots & 1 \\
& & & \ddots & \ddots & 1 \\
& & & & -1 & 1
\end{array}\right] .
$$

Table 1(a) and (b) show the numerical results for $A_{n}$ using MINRES or GMRES. The preconditioners seem efficient for speeding up the rate of convergence of both Krylov subspace methods. 
Table 1 Numbers of iterations with (a) MINRES for $Y_{n} A_{n}$ and (b) GMRES for $A_{n}$ with Grcar matrix $A_{n}$

(a)

\begin{tabular}{|l|c|c|}
\hline$n$ & $I_{n}$ & $\left|c\left(A_{n}\right)\right|$ \\
\hline 128 & 49 & 13 \\
256 & 49 & 12 \\
512 & 49 & 11 \\
1024 & 47 & 11 \\
\hline
\end{tabular}

(b)

\begin{tabular}{|l|c|c|}
\hline$n$ & $I_{n}$ & $c\left(A_{n}\right)$ \\
\hline 128 & 94 & 6 \\
256 & 158 & 6 \\
512 & 218 & 6 \\
1024 & 213 & 5 \\
\hline
\end{tabular}

Example 2. We now consider $h\left(A_{n}\right)$ with $h(z)=z^{2}+z+1$ and $A_{n}$ being a Grcar matrix. Table 2 (a) and (b) show the numerical results for $h\left(A_{n}\right)$. In Figure 1 (a) and (b), we also show the spectra of $Y_{n} h\left(A_{n}\right)$ before or after applying the preconditioner $\left|h\left(c\left(A_{n}\right)\right)\right|$ when $n=512$. Again, we observe the clusters of eigenvalues at \pm 1 .

Table 2 Numbers of iterations with (a) MINRES for $Y_{n} h\left(A_{n}\right)$ and (b) GMRES for $h\left(A_{n}\right)$ with $h(z)=z^{2}+z+1$ and $A_{n}$ being a Grcar matrix.

(a)

\begin{tabular}{|l|c|c|}
\hline$n$ & $I_{n}$ & $\left|h\left(c\left(A_{n}\right)\right)\right|$ \\
\hline 128 & 144 & 16 \\
256 & 167 & 15 \\
512 & 194 & 14 \\
1024 & 190 & 13 \\
\hline
\end{tabular}

(b)

\begin{tabular}{|l|c|c|}
\hline$n$ & $I_{n}$ & $h\left(c\left(A_{n}\right)\right)$ \\
\hline 128 & 128 & 9 \\
256 & 256 & 8 \\
512 & 512 & 8 \\
1024 & 1024 & 7 \\
\hline
\end{tabular}




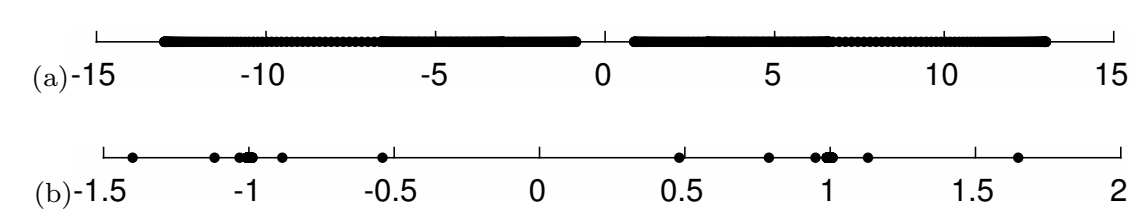

Fig. 1 The spectrum of $Y_{n} h\left(A_{n}\right)$ when $n=512$ with $h(z)=z^{2}+z+1$ and $A_{n}$ being a Grcar matrix under the following condition: (a) without a preconditioner; (b) with the preconditioner $\left|h\left(c\left(A_{n}\right)\right)\right|$.

Example 3. We also consider the system defined by hyperbolic sine function of Toeplitz matrix. Table 3 (a) and (b) show the numerical results for $\sinh A_{n}$ with $A_{n}$ being Grcar matrix. The convergence rate appears accelerated with our proposed preconditioners.

Table 3 Numbers of iterations with (a) MINRES for $Y_{n} \sinh A_{n}$ and (b) GMRES for $\sinh A_{n}$ with $A_{n}$ being a Grcar matrix.

(a)

\begin{tabular}{|l|c|c|}
\hline$n$ & $I_{n}$ & $\left|\sinh c\left(A_{n}\right)\right|$ \\
\hline 64 & 105 & 24 \\
128 & 172 & 22 \\
256 & 391 & 20 \\
512 & 808 & 19 \\
\hline
\end{tabular}

(b)

\begin{tabular}{|l|c|c|}
\hline$n$ & $I_{n}$ & $\sinh c\left(A_{n}\right)$ \\
\hline 64 & 64 & 14 \\
128 & 124 & 13 \\
256 & 240 & 11 \\
512 & 486 & 10 \\
\hline
\end{tabular}


Example 4. The next example is the (real) nonsymmetric Toeplitz matrix generated by $f(\theta)=e^{i \theta}+2 e^{-i \theta}$, namely

$$
A_{n}=\left[\begin{array}{lll} 
& 2 & \\
1 & & \ddots \\
& \ddots & \\
& & 1
\end{array}\right] .
$$

In Figure 2, we still observe that the eigenvalues are highly clustered at \pm 1 as expected.

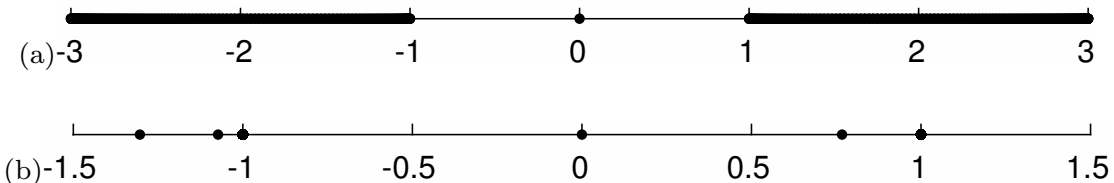

Fig. 2 The spectrum of $Y_{n} A_{n}$ with $A_{n}$ generated by $f(\theta)=e^{i \theta}+2 e^{-i \theta}$ when $n=512$ under the following condition: (a) without a preconditioner; (b) with the preconditioner $\left|c\left(A_{n}\right)\right|$. 
Example 5. We consider the matrix exponentials with $A_{n}$ generated by $f(\theta)=\theta^{2}$. Table 4 shows the numbers of iterations required with CG for $e^{A_{n}}$ with or without the preconditioner. It is apparent that the proposed preconditioners are effective for speeding up the rate of convergence of CG.

Table 4 Numbers of iterations with CG for $e^{A_{n}}$ with $A_{n}$ generated by $f(\theta)=\theta^{2}$.

\begin{tabular}{|l|c|c|}
\hline$n$ & $I_{n}$ & $e^{c\left(A_{n}\right)}$ \\
\hline 128 & 258 & 9 \\
256 & 465 & 8 \\
512 & 673 & 8 \\
1024 & 835 & 8 \\
\hline
\end{tabular}

In Figure 3, we further show the spectra of the preconditioned matrices with different $n$. We observe that the highly clustered spectrum is independent of $n$. In Figure 3 (i) and (ii), the contrast between the spectra of the systems is shown. In Figure 3 (iii), we show the zoom-in spectrum of (ii) and observe that the eigenvalues are highly clustered at 1 . Due to the highly clustered eigenvalues of the preconditioned system, a fast convergence rate for $\mathrm{CG}$ is expected (see for example [1]). 
(a) (i) $0 \quad 2000 \quad 4000 \quad 6000 \quad 8000 \quad 10000 \quad 12000 \quad 1400016000 \quad 18000$ (ii)

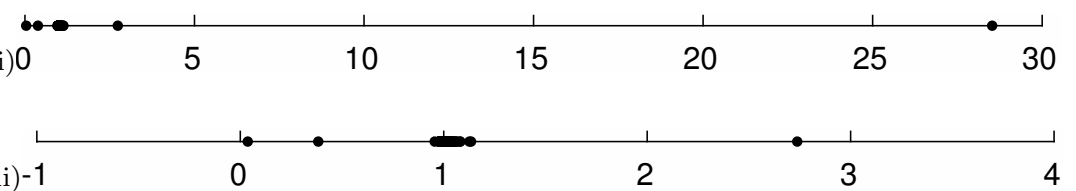

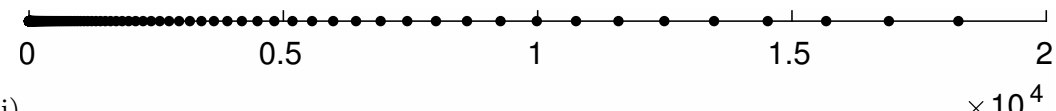

(b) (i)

$\times 10^{4}$

(ii) 0

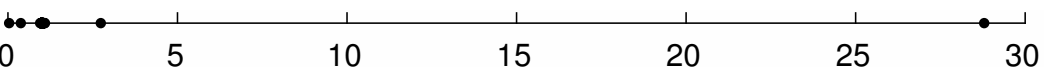

(iii)-1

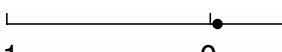

1

2

3

4

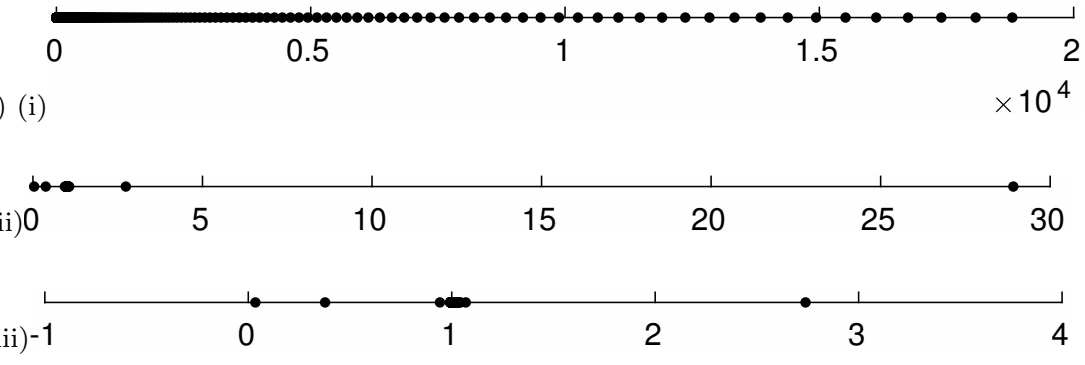

Fig. 3 The spectrum of $e^{A_{n}}$ with $A_{n}$ generated by $f(\theta)=\theta^{2}$ when (a) $n=128$, (b) $n=256$ or (c) $n=512$ under the following condition: (i) without a preconditioner; (ii) with the preconditioner $e^{c\left(A_{n}\right)}$. (iii) The zoom-in spectrum of (ii). 
Example 6. Next, we consider symmetric indefinite Toeplitz matrices. Table 5 shows the numerical results for $A_{n}$ generated by $f(\theta)=\theta^{2}-\pi$. In Figure 4 (a) and (b), we also show the spectra of the system before and after applying the preconditioners. We observe the clusters of eigenvalues at \pm 1 which are independent of $n$.

Table 5 Numbers of iterations with (a) MINRES and (b) GMRES for $A_{n}$ with $A_{n}$ generated by $f(\theta)=\theta^{2}-\pi$.

(a)

\begin{tabular}{|l|c|c|}
\hline$n$ & $I_{n}$ & $\left|c\left(A_{n}\right)\right|$ \\
\hline 128 & 76 & 11 \\
256 & 159 & 11 \\
512 & 321 & 10 \\
1024 & 648 & 10 \\
\hline
\end{tabular}

(b)

\begin{tabular}{|l|c|c|}
\hline$n$ & $I_{n}$ & $c\left(A_{n}\right)$ \\
\hline 128 & 74 & 6 \\
256 & 150 & 7 \\
512 & 299 & 6 \\
1024 & 595 & 6
\end{tabular}



(a) (i) -4
0 2
4
6
8
(ii) -5

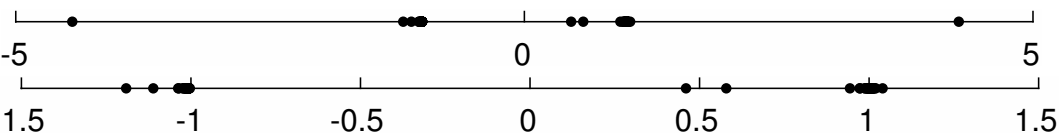

(b) (i) -4

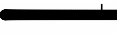

2

4

6

8

(ii) -3

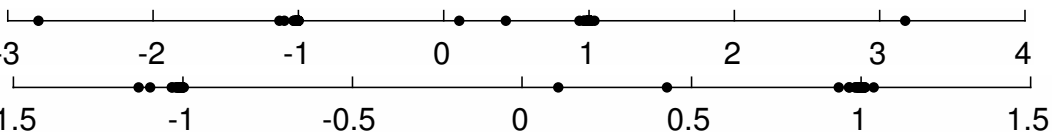

(c) (i) -4

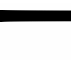

(ii)

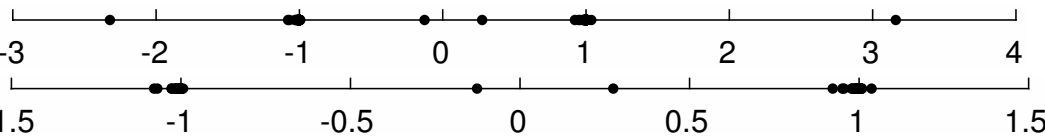

(iii) -1.5

Fig. 4 (i) The spectrum of $A_{n}$ and that of with $A_{n}$ is generated by $f(\theta)=\theta^{2}-\pi$ when (a) $n=128$, (b) $n=256$ or (c) $n=512$ under the following condition: (i) without a preconditioner; (ii) with the preconditioner $\left|c\left(A_{n}\right)\right|$. (iii) The zoom-in spectrum of (ii). 
Example 7. In this case, we consider $\cos A_{n}$ with the symmetric indefinite $A_{n}$ generated by $f(\theta)=\theta^{2}-\pi$. Table 6 shows the numerical results with MINRES for $\cos A_{n}$ with or without preconditioners $\left|\cos c\left(A_{n}\right)\right|$. Again, we observe significant reduce in the numbers of iterations needed for MINRES with the proposed preconditioners used. In Figure 5, we also show the spectra of $\left|\cos c\left(A_{n}\right)\right|^{-1} \cos A_{n}$ when $n=512$ and observe the clusters of eigenvalues at \pm 1 .

Table 6 Numbers of iterations with (a) MINRES and (b) GMRES for $\cos A_{n}$ with $A_{n}$ generated by $f(\theta)=\theta^{2}-\pi$.

(a)

\begin{tabular}{|l|c|c|}
\hline$n$ & $I_{n}$ & $\left|\cos c\left(A_{n}\right)\right|$ \\
\hline 128 & 58 & 24 \\
256 & 110 & 24 \\
512 & 212 & 24 \\
1024 & 417 & 22 \\
\hline
\end{tabular}

(b)

\begin{tabular}{|l|c|c|}
\hline$n$ & $I_{n}$ & $\cos c\left(A_{n}\right)$ \\
\hline 128 & 58 & 12 \\
256 & 110 & 13 \\
512 & 212 & 11 \\
1024 & 417 & 11 \\
\hline
\end{tabular}

(a) -1

$-0.5$

0

0.5

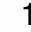

(b) -40

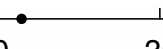

$-30$

$-20$

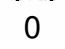

20

(b) -1.5

$-1$

$-0.5$

0.5

1.5

Fig. 5 The spectrum of $\cos A_{n}$ when $n=512$ with $A_{n}$ generated by $f(\theta)=\theta^{2}-\pi$ under the following condition: (a) without a preconditioner; (b) with the preconditioner $\left|\cos c\left(A_{n}\right)\right|$. (c) The zoom-in spectrum of (b). 
We recall that the cost of GMRES increases for every iteration, whereas MINRES has a constant cost per iteration. Thus whilst in many examples, GMRES requires fewer iterations than MINRES, there is not necessarily a reduction in work. The rapid convergence guarantees established here for MINRES are a true indication of the success of our preconditioning approach; it is not perhaps surprising that related preconditioning strategies such as those we have used with GMRES are also successful. It is now widely appreciated that a good preconditioner will lead to work well with efficient iterative methods; indeed that it is the quality of the preconditioner rather than the choice of iterative method which usually determines the success or otherwise of a solver.

\section{References}

1. Owe Axelsson and Gunhild Lindskog. On the rate of convergence of the preconditioned conjugate gradient method. Numerische Mathematik, 48:499-524, 1986.

2. D.A. Bini, S. Dendievel, G. Latouche, and B. Meini. Computing the exponential of large block-triangular block-Toeplitz matrices encountered in fluid queues. Linear Algebra and its Applications, 502(Supplement C):387 - 419, 2016. Structured Matrices: Theory and Applications.

3. Raymond H. Chan and Man-Chung Yeung. Circulant preconditioners for complex Toeplitz matrices. SIAM Journal on Numerical Analysis, 30(4):1193-1207, 1993.

4. T. F. Chan. An optimal circulant preconditioner for Toeplitz systems. SIAM Journal on Scientific and Statistical Computing, 9(4):766-771, 1988.

5. D.J. Duffy. Finite Difference Methods in Financial Engineering: A Partial Differential Equation Approach. The Wiley Finance Series. Wiley, 2013.

6. Nicholas J. Higham. Functions of matrices. Society for Industrial and Applied Mathematics (SIAM), Philadelphia, PA, 2008. Theory and computation.

7. Nicholas J. Higham and Peter Kandolf. Computing the action of trigonometric and hyperbolic matrix functions. SIAM Journal on Scientific Computing, 39(2):A613-A627, 2017.

8. Sean Hon. Optimal preconditioners for systems defined by functions of Toeplitz matrices. Under review.

9. D. Kressner and R. Luce. Fast computation of the matrix exponential for a Toeplitz matrix. ArXiv e-prints, July 2016.

10. Spike T. Lee, Hong-Kui Pang, and Hai-Wei Sun. Shift-invert Arnoldi approximation to the Toeplitz matrix exponential. SIAM Journal on Scientific Computing, 32(2):774$792,2010$.

11. Eleanor McDonald, Sean Hon, Jennifer Pestana, and Andy Wathen. Preconditioning for Nonsymmetry and Time-Dependence, pages 81-91. Springer International Publishing, Cham, 2017.

12. Michael K. Ng. Iterative methods for Toeplitz systems. Numerical Mathematics and Scientific Computation. Oxford University Press, New York, 2004.

13. Michael K. Ng and Jianyu Pan. Approximate inverse circulant-plus-diagonal preconditioners for Toeplitz-plus-diagonal matrices. SIAM Journal on Scientific Computing, 32(3):1442-1464, 2010.

14. J. Pestana and A. J. Wathen. A preconditioned MINRES method for nonsymmetric Toeplitz matrices. SIAM Journal on Matrix Analysis and Applications, 36(1):273-288, 2015.

15. E.W. Sachs and A.K. Strauss. Efficient solution of a partial integro-differential equation in finance. Applied Numerical Mathematics, 58(11):1687 - 1703, 2008. 